\title{
A PD-type Regulator with Exact Gravity Cancellation for Robots with Flexible Joints
}

\author{
Alessandro De Luca
}

\begin{abstract}
We present a new control approach to regulation tasks for robots with elastic joints in the presence of gravity. The control law combines a term that cancels the gravity effects on the robot link dynamics with a PD-type error feedback on the motor variables. The first control component follows from the feedback equivalence principle when imposing to the link variables the same dynamic behavior as if gravity were absent. The PD component can then be designed in a rather straightforward way. Global asymptotic stability is shown via Lyapunov analysis, without the need of strictly positive lower bounds neither on the proportional control gain nor on the structural joint stiffness. The control approach is also extended to the case of robot joints with nonlinear stiffness.
\end{abstract}

\section{INTRODUCTION}

Set-point regulation of robots is usually achieved by a linear, decentralized PD control law at the joints with some form of gravity cancellation or compensation [1]. Perfect cancellation of gravity is trivial for fully rigid manipulators. In fact, for the standard dynamic model

$$
\boldsymbol{M}(\boldsymbol{q}) \ddot{\boldsymbol{q}}+\boldsymbol{C}(\boldsymbol{q}, \dot{\boldsymbol{q}}) \dot{\boldsymbol{q}}+\boldsymbol{g}(\boldsymbol{q})=\boldsymbol{\tau},
$$

the choice

$$
\boldsymbol{\tau}=\boldsymbol{\tau}_{g}+\boldsymbol{\tau}_{0}, \quad \boldsymbol{\tau}_{g}=\boldsymbol{g}(\boldsymbol{q})
$$

removes gravity from the picture (both statically and dynamically), thanks to the collocation of gravity and input torques (and full actuation of the system). For regulation tasks to a constant $\boldsymbol{q}_{d}$, it is sufficient to set $\boldsymbol{\tau}_{0}=\boldsymbol{K}_{P}\left(\boldsymbol{q}_{d}-\boldsymbol{q}\right)-\boldsymbol{K}_{D} \dot{\boldsymbol{q}}$, with (diagonal) positive definite gain matrices $\boldsymbol{K}_{P}$ and $\boldsymbol{K}_{D}$. Global regulation can also be obtained with a gravity compensation scheme, where $\boldsymbol{\tau}_{g}=\boldsymbol{g}\left(\boldsymbol{q}_{d}\right)$ is the constant gravity term at the desired configuration $\boldsymbol{q}_{d}$ and $\boldsymbol{\tau}_{0}$ is again a PD control law. The only additional requirement is that the proportional gain $\boldsymbol{K}_{P}$ should dominate the norm of the gradient of the gravity vector.

Here we are interested in the problem of global regulation of robots with flexible joints under the weakest possible control assumptions. One main motivation comes from the domain of physical Human-Robot Interaction (pHRI), where achieving zero-gravity operation [2] avoids biasing the robot reaction to collisions along the gradient of the gravitational potential, with a safer robot behavior in its whole workspace [3]. Robots intended for pHRI have lightweight but rigid links, and include compliant elements in their mechanical construction in order to reduce the possibility of injuries due to unexpected collisions. Compliance is typically

The authors are with the Dipartimento di Informatica e Sistemistica, Università di Roma "La Sapienza", Via Ariosto 25, 00185 Rome, Italy \{deluca,fflacco\}@dis.uniroma1.it

\author{
Fabrizio Flacco
}

concentrated in the transmissions at the joints, by using either harmonic drives with constant or nonlinear stiffness [4], [5] or variable and independently actuated nonlinear stiffness [6], [7].

We shall consider robot manipulators having $n$ elastic joints of constant stiffness and $n$ driving motors. Let $\boldsymbol{q}$ and $\boldsymbol{\theta}$ be the $n$-dimensional vectors of link and motor variables. Under the simplifying modeling assumption of Spong [8], the dynamic model takes the form

$$
\begin{aligned}
\boldsymbol{M}(\boldsymbol{q}) \ddot{\boldsymbol{q}}+\boldsymbol{C}(\boldsymbol{q}, \dot{\boldsymbol{q}}) \dot{\boldsymbol{q}}+\boldsymbol{g}(\boldsymbol{q})+\boldsymbol{K}(\boldsymbol{q}-\boldsymbol{\theta}) & =\mathbf{0} \\
\boldsymbol{B} \ddot{\boldsymbol{\theta}}+\boldsymbol{K}(\boldsymbol{\theta}-\boldsymbol{q}) & =\boldsymbol{\tau},
\end{aligned}
$$

where $\boldsymbol{M}>0$ is the robot inertia matrix, the diagonal matrix $\boldsymbol{B}>0$ contains the motor inertias (as reflected through the gear ratios), $\boldsymbol{C} \dot{\boldsymbol{q}}$ are the centrifugal and Coriolis terms, $\boldsymbol{g}$ is the gravity vector, and $\boldsymbol{K}>0$ is the diagonal matrix of constant joint stiffnesses. In (1-2), the actuation torques $\tau$ appear on the motor side of the elastic joints, while gravity loading $\boldsymbol{g}(\boldsymbol{q})$ affects primarily the dynamic behavior of the link variables. This non-colocation is a major problem for control. For regulation tasks, when the link position $\boldsymbol{q}$ has to be asympotically stabilized to a desired constant value $\boldsymbol{q}_{d}$, different gravity compensation laws have been proposed. A first solution is based on motor PD feedback with constant gravity compensation [9]

$$
\boldsymbol{\tau}_{0}=\boldsymbol{K}_{P}\left(\boldsymbol{\theta}_{d}-\boldsymbol{\theta}\right)-\boldsymbol{K}_{D} \dot{\boldsymbol{\theta}}, \quad \boldsymbol{\tau}_{g}=\boldsymbol{g}\left(\boldsymbol{q}_{d}\right),
$$

where $\boldsymbol{\theta}_{d}=\boldsymbol{q}_{d}+\boldsymbol{K}^{-1} \boldsymbol{g}\left(\boldsymbol{q}_{d}\right)$ and $\boldsymbol{K}_{D}>0$. Indeed, this compensation cancels gravity only in the final steadystate condition. In order to show global asymptotic stability by Lyapunov arguments, the proportional gain matrix $\boldsymbol{K}_{P}$ should be chosen larger than a positive constant related to gravity - similarly to the rigid case. This can always be achieved, provided that the joint stiffness matrix $\boldsymbol{K}$ dominates in turn the gradient of $\boldsymbol{g}(\boldsymbol{q})$ (a structural assumption). In order to improve the transient performance, an on-line compensation has been proposed in [10], evaluating $\boldsymbol{g}$ in $\boldsymbol{\tau}_{g}$ with a gravity-biased measure of the motor position

$$
\boldsymbol{\tau}_{g}=\boldsymbol{g}(\tilde{\boldsymbol{\theta}}), \quad \tilde{\boldsymbol{\theta}}=\boldsymbol{\theta}-\boldsymbol{K}^{-1} \boldsymbol{g}\left(\boldsymbol{q}_{d}\right) .
$$

However, the theoretical restrictions on $\boldsymbol{K}_{P}$ and $\boldsymbol{K}$ could not be relaxed in the analysis. A better result was achieved in [11], with a gravity compensation term of the form

$$
\boldsymbol{\tau}_{g}=\boldsymbol{g}(\overline{\boldsymbol{q}}(\boldsymbol{\theta}))
$$

where $\overline{\boldsymbol{q}}(\boldsymbol{\theta})$ is computed by numerically solving the quasistatic relation $\boldsymbol{g}(\boldsymbol{q})+\boldsymbol{K}(\boldsymbol{q}-\boldsymbol{\theta})=\mathbf{0}$, for any measured motor 
position $\boldsymbol{\theta}$. This variant eliminates the need of a strictly positive lower bound on $\boldsymbol{K}_{P}$ and asymptotic stability can be shown through a modified Lyapunov function. Nonetheless, the structural assumption concerning the joint stiffness should still hold.

All the above control laws have the merit of using only feedback from the motor variables $\boldsymbol{\theta}$ and $\dot{\boldsymbol{\theta}}$. A practical solution for compensating gravity in elastic joint robots has been proposed also in [12], based on the availability of joint torque sensors. The use of this additional sensor can be interpreted as involving also the link position $\boldsymbol{q}$ and its derivative in the control law. On the other hand, none of the control laws proposed so far is able to remove completely the effects of gravity, especially in highly dynamic tasks: only a partial compensation, and not a cancellation, of the gravitational load acting on the robot link motion is obtained. In the context of robot reaction to unintended collisions, dynamic operation is the most critical condition, e.g., for an impact occurring during the transient phase of a fast regulation task. Therefore, the possibility of shaping the robot response by getting rid in full of the gravity effects on the moving links is an interesting feature.

In this paper, we present a global regulation control law for robots with flexible joints that addresses the above open problems. The state feedback controller includes a gravity cancellation term so as to accurately match, in any dynamic condition, the behavior of the links as if they were moving in the absence of gravity (Sect. II). This result is obtained using the general principle of feedback equivalence [13]. It is well known that all robots with elastic joints modeled by (1-2) can be exactly linearized by means of a static state feedback [8]. This control property is used to derive the dynamic gravity cancellation torque $\tau_{g}$, without resorting to the full complexity of a feedback linearization control law. On top of this, a PD-type law is defined for the stabilizing control term $\tau_{0}$ and global asymptotic stability is proven via the Lyapunov technique (Sect. III and Appendix). Illustrative simulation results are reported in Sect. IV. Finally, the analysis is extended to the case of joints with nonlinear flexibility in Sect. V.

\section{CAncellation of GRAVity in Robots With ELASTIC JOINTS}

Our control goal is to define a nonlinear state feedback law $\boldsymbol{\tau}=\boldsymbol{\tau}\left(\boldsymbol{q}, \boldsymbol{\theta}, \dot{\boldsymbol{q}}, \dot{\boldsymbol{\theta}}, \boldsymbol{\tau}_{0}\right)$ in (2) such that the behavior of the compensated system matches in suitable coordinates the one of an equivalent model without gravity, i.e.,

$$
\begin{aligned}
\boldsymbol{M}\left(\boldsymbol{q}_{0}\right) \ddot{\boldsymbol{q}}_{0}+\boldsymbol{C}\left(\boldsymbol{q}_{0}, \dot{\boldsymbol{q}}_{0}\right) \dot{\boldsymbol{q}}_{0}+\boldsymbol{K}\left(\boldsymbol{q}_{0}-\boldsymbol{\theta}_{0}\right) & =\mathbf{0} \\
\boldsymbol{B} \ddot{\boldsymbol{\theta}}_{0}+\boldsymbol{K}\left(\boldsymbol{\theta}_{0}-\boldsymbol{q}_{0}\right) & =\boldsymbol{\tau}_{0},
\end{aligned}
$$

where a subscript 0 characterizes the variables of the robot in the absence of gravity.

System (1-2) with gravity and system (3-4) without gravity are both exactly linearizable by means of a static state feedback into decoupled chains of four integrators, with the link positions and their first three time derivatives being the linearizing coordinates. Therefore, thanks to the feedback equivalence principle, imposing the equality

$$
\boldsymbol{q}(t) \equiv \boldsymbol{q}_{0}(t), \quad \forall t \geq 0,
$$

we should obtain the desired result. The validity of this simple idea can be verified as follows.

Differentiating eq. (1) w.r.t. time yields

$$
\begin{aligned}
\boldsymbol{M}(\boldsymbol{q}) \boldsymbol{q}^{[3]}+(\dot{\boldsymbol{M}}(\boldsymbol{q})+\boldsymbol{C}(\boldsymbol{q}, \dot{\boldsymbol{q}})) \ddot{\boldsymbol{q}}+\dot{\boldsymbol{C}}(\boldsymbol{q}, \dot{\boldsymbol{q}}) \dot{\boldsymbol{q}} \\
+\dot{\boldsymbol{g}}(\boldsymbol{q})+\boldsymbol{K}(\dot{\boldsymbol{q}}-\dot{\boldsymbol{\theta}})=\mathbf{0}
\end{aligned}
$$

with the notation $\boldsymbol{q}^{[i]}=d^{i} \boldsymbol{q} / d t^{i}$. Differentiating once more and substituting $\ddot{\boldsymbol{\theta}}$ from (2), we obtain

$$
\boldsymbol{f}\left(\boldsymbol{q}, \dot{\boldsymbol{q}}, \ddot{\boldsymbol{q}}, \boldsymbol{q}^{[3]}, \boldsymbol{q}^{[4]}\right)=\boldsymbol{K} \boldsymbol{B}^{-1}(\boldsymbol{\tau}-\boldsymbol{K}(\boldsymbol{\theta}-\boldsymbol{q}))-\ddot{\boldsymbol{g}}(\boldsymbol{q}),
$$

where

$$
\begin{aligned}
\boldsymbol{f}= & \boldsymbol{M}(\boldsymbol{q}) \boldsymbol{q}^{[4]}+(2 \dot{\boldsymbol{M}}(\boldsymbol{q})+\boldsymbol{C}(\boldsymbol{q}, \dot{\boldsymbol{q}})) \boldsymbol{q}^{[3]} \\
& +(\ddot{\boldsymbol{M}}(\boldsymbol{q})+2 \dot{\boldsymbol{C}}(\boldsymbol{q}, \dot{\boldsymbol{q}})+\boldsymbol{K}) \ddot{\boldsymbol{q}}+\ddot{\boldsymbol{C}}(\boldsymbol{q}, \dot{\boldsymbol{q}}) \dot{\boldsymbol{q}}
\end{aligned}
$$

is a function of $\boldsymbol{q}$ and its first four derivatives only. Repeating the same derivation on the gravity-free model (3-4) leads to

$$
\boldsymbol{f}\left(\boldsymbol{q}_{0}, \dot{\boldsymbol{q}}_{0}, \ddot{\boldsymbol{q}}_{0}, \boldsymbol{q}_{0}^{[3]}, \boldsymbol{q}_{0}^{[4]}\right)=\boldsymbol{K} \boldsymbol{B}^{-1}\left(\boldsymbol{\tau}_{0}-\boldsymbol{K}\left(\boldsymbol{\theta}_{0}-\boldsymbol{q}\right)\right) .
$$

By imposing (5), the left-hand sides of (6) and (7) are equal, and thus

$$
\boldsymbol{\tau}-\boldsymbol{K}(\boldsymbol{\theta}-\boldsymbol{q})-\boldsymbol{B} \boldsymbol{K}^{-1} \ddot{\boldsymbol{g}}(\boldsymbol{q})=\boldsymbol{\tau}_{0}-\boldsymbol{K}\left(\boldsymbol{\theta}_{0}-\boldsymbol{q}\right) .
$$

To eliminate from (8) the motor variable $\boldsymbol{\theta}_{0}$ of the gravityfree model, we equate the left-hand sides of (1) and (3), and use again (5). This yields

$$
\boldsymbol{\theta}_{0}=\boldsymbol{\theta}-\boldsymbol{K}^{-1} \boldsymbol{g}(\boldsymbol{q})
$$

Replacing eq. (9) into (8), the solution of our problem is given by the control law

$$
\tau=\tau_{g}+\tau_{0}
$$

with

$$
\boldsymbol{\tau}_{g}=\boldsymbol{g}(\boldsymbol{q})+\boldsymbol{B} \boldsymbol{K}^{-1} \ddot{\boldsymbol{g}}(\boldsymbol{q})
$$

where

$$
\begin{aligned}
\ddot{\boldsymbol{g}}(\boldsymbol{q})= & \frac{\partial \boldsymbol{g}(\boldsymbol{q})}{\partial \boldsymbol{q}} \boldsymbol{M}^{-1}(\boldsymbol{q})(\boldsymbol{K}(\boldsymbol{\theta}-\boldsymbol{q})-\boldsymbol{C}(\boldsymbol{q}, \dot{\boldsymbol{q}}) \dot{\boldsymbol{q}}-\boldsymbol{g}(\boldsymbol{q})) \\
& +\sum_{i=1}^{n} \frac{\partial^{2} \boldsymbol{g}(\boldsymbol{q})}{\partial \boldsymbol{q} \partial q_{i}} \dot{\boldsymbol{q}} \dot{q}_{i} .
\end{aligned}
$$

As a result, under the action of the control law (10-11), the link evolution of the gravity-loaded system (1-2), for all times $t \geq 0$ and any command $\tau_{0}$, will be the same as that of the gravity-free system (3-4) initialized at

$$
\begin{aligned}
\boldsymbol{q}_{0}(0) & =\boldsymbol{q}(0) & \dot{\boldsymbol{q}}_{0}(0) & =\dot{\boldsymbol{q}}(0) \\
\ddot{\boldsymbol{q}}_{0}(0) & =\ddot{\boldsymbol{q}}(0) & \boldsymbol{q}_{0}^{[3]}(0) & =\boldsymbol{q}^{[3]}(0) .
\end{aligned}
$$

In static conditions $(\dot{\boldsymbol{q}}=\ddot{\boldsymbol{q}}=\mathbf{0})$, the gravity cancellation torque (11) becomes $\boldsymbol{\tau}_{g}=\boldsymbol{g}(\boldsymbol{q})$ as expected. Instead, in dynamic conditions $\tau_{g}$ includes a term that is proportional to the inverse of the joint stiffness $\boldsymbol{K}$. Thus, the more rigid are the transmissions the less extra dynamic torque is needed for 
gravity cancellation. For $\boldsymbol{K} \rightarrow \infty$, we recover the standard gravity cancellation torque of the rigid case.

It should be stressed that, despite of the need of inverting the robot inertia matrix $\boldsymbol{M}(\boldsymbol{q})$, the gravity cancellation torque (11) is much simpler than the expression of a feedback linearization control law, which involves also the time derivatives of the model terms $\boldsymbol{M}(\boldsymbol{q})$ and $\boldsymbol{C}(\boldsymbol{q}, \dot{\boldsymbol{q}})$ up to the second order [14].

Finally, we note that there are still differences in the state behavior between the gravity-free system and the original gravity-loaded system under the gravity cancellation control law (10-11). While the two systems will evolve in an identical way when looking at the linearizing link coordinates, the inverse mappings of this evolution in terms of the respective motor variables will be different, as dictated by eq. (9). This should not be surprising from a physical point of view: the gravity-loaded robot needs the presence of a deformation $\boldsymbol{q}-\boldsymbol{\theta} \neq \boldsymbol{q}-\boldsymbol{\theta}_{0}$ that balances the gravity on the link side. The control law (10-11) will only cancel the dynamic effects of gravity on link motion, while the term $\tau_{0}$ in (10) is left for further control design.

\section{A PD-TYPE REGULATOR FOR RoBots WITH ELASTIC JOINTS}

Consider again the gravity-loaded system (1-2). We address the problem of asymptotic stabilization of a desired (closed-loop) equilibrium state

$$
\boldsymbol{q}=\boldsymbol{q}_{d}, \quad \boldsymbol{\theta}=\boldsymbol{\theta}_{d}:=\boldsymbol{q}_{d}+\boldsymbol{K}^{-1} \boldsymbol{g}\left(\boldsymbol{q}_{d}\right), \quad \dot{\boldsymbol{q}}=\dot{\boldsymbol{\theta}}=\mathbf{0}
$$

The desired motor position $\boldsymbol{\theta}_{d}$ is dictated by static analysis (for $\dot{\boldsymbol{q}}=\ddot{\boldsymbol{q}}=\mathbf{0}$ ) of eq. (1) at the desired link position $\boldsymbol{q}_{d}$.

Taking advantage of the dynamic gravity cancellation law (11), a regulation controller realizing the task can be defined as

$$
\boldsymbol{\tau}=\boldsymbol{\tau}_{g}+\boldsymbol{\tau}_{0}
$$

where $\tau_{g}$ is given by (11) and $\tau_{0}$ is the PD-type law

$$
\boldsymbol{\tau}_{0}=\boldsymbol{K}_{P}\left(\boldsymbol{q}_{d}-\boldsymbol{\theta}+\boldsymbol{K}^{-1} \boldsymbol{g}(\boldsymbol{q})\right)-\boldsymbol{K}_{D}\left(\dot{\boldsymbol{\theta}}-\boldsymbol{K}^{-1} \dot{\boldsymbol{g}}(\boldsymbol{q})\right) .
$$

The following result holds.

Theorem 1: The desired state (13) is the unique equilibrium state for closed-loop system (1-2) with control law (1415). Moreover, if

$$
\boldsymbol{K}_{P}>0, \quad \boldsymbol{K}_{D}>0,
$$

the desired state is globally asymptotically stable.

The proof of Theorem 1 is given in the Appendix. Some remarks are in order:

- The stabilizing control law (15) is logically derived from a pure PD scheme on the motor variables $\boldsymbol{\theta}_{0}$ and $\dot{\boldsymbol{\theta}}_{0}$ of the gravity-free system,

$$
\begin{aligned}
\boldsymbol{\tau}_{0} & =\boldsymbol{K}_{P}\left(\boldsymbol{\theta}_{d 0}-\boldsymbol{\theta}_{0}\right)-\boldsymbol{K}_{D} \dot{\boldsymbol{\theta}}_{0} \\
& =\boldsymbol{K}_{P}\left(\boldsymbol{q}_{d}-\boldsymbol{\theta}+\boldsymbol{K}^{-1} \boldsymbol{g}(\boldsymbol{q})\right)-\boldsymbol{K}_{D}\left(\dot{\boldsymbol{\theta}}-\boldsymbol{K}^{-1} \dot{\boldsymbol{g}}(\boldsymbol{q})\right),
\end{aligned}
$$

using the relation (9) between motor positions of the gravity-free system and of the gravity-loaded system under the dynamic gravity cancellation torque (similarly for motor velocities). The motor reference for the PD is $\boldsymbol{\theta}_{d 0}=\boldsymbol{q}_{d}$, since gravitational effects on the links have been canceled by $\tau_{g}$.

- As stated by Theorem 1 , there is no need of a strictly positive lower bound on the joint stiffness $\boldsymbol{K}$, contrary to all previous literature [9]-[11]. While joint stiffness typically dominates the gradient of gravity torques in industrial robots with elastic joints, this relaxation can be of interest for systems with variable stiffness actuation, where very low stiffness values are desirable for limiting injuries due to collisions.

- Both the PD term (15) and $\tau_{g}$ require feedback from the full state of the robot. While this is the same requisite of a feedback linearization law, gravity is eliminated here without a complete cancellation of the dynamics of the elastic joint robot, which is considerably simpler.

- From the proof of Theorem 1, it is easy to see that the desired state would still be the unique equilibrium for the closed-loop system when reducing $\tau_{g}$ just to $\boldsymbol{g}(\boldsymbol{q})$. However, a proof of global asymptotic stability with such a reduced term added to a PD law is not available.

- The control law (14-15) bears strong similarities with the one independently obtained in [15, p. 119], which involves also a motor inertia shaping and is derived from a passivity approach. However, the present framework allows to explicitly discard the structural assumption on $\boldsymbol{K}$ and provides the more general feature of exact reproduction of link motion as in the gravity-free case.

\section{Simulation Results}

To illustrate the typical behavior obtained under the action of the PD-type control law (15) with dynamic gravity cancellation (11), it is sufficient to consider a single link with an elastic joint in the absence of gravity and under gravity. In this case, the link inertia is a constant scalar $M$ and the gravity term is $g(q)=m d g_{0} \sin q$, where $m$ is the mass of the link, $d$ the distance of its center of mass from the joint, and $g_{0}$ the gravity acceleration. The term $\tau_{g}$ is

$$
\begin{aligned}
\tau_{g}=m d g_{0}\{ & \left(1-\frac{B}{K} \dot{q}^{2}\right) \sin q-\frac{B}{M} \frac{m d g_{0}}{K} \sin q \cos q \\
& \left.+\frac{B}{M}(\theta-q) \cos q\right\} .
\end{aligned}
$$

while the PD term $\tau_{0}$ is

$$
\tau_{0}=K_{P}\left(q_{d}-\theta+\frac{m d g_{0}}{K} \sin q\right)-K_{D}\left(\dot{\theta}-\frac{m d g_{0}}{K} \cos q \dot{q}\right)
$$

Using as data $M=B=0.9333\left[\mathrm{~N} \cdot \mathrm{m} \cdot \mathrm{s}^{2} / \mathrm{rad}\right], m=10[\mathrm{~kg}]$, $d=0.25[\mathrm{~m}]$, and $K=100[\mathrm{~N} \cdot \mathrm{m} / \mathrm{rad}]$, we simulated the two systems starting at rest from the downward equilibrium $q=\theta=0$. The link position is commanded to $q_{d}=\pi / 2$. The PD gains were chosen as $K_{P}=25$ and $K_{D}=10$. These values were conveniently tuned in the absence of gravity.

Figure 1 shows the evolution of the relevant variables in the two cases of no gravity and presence of gravity with its dynamic cancellation. The link motion in Fig. 1(a) is exactly the same (plots are superposed). The motor position has a 

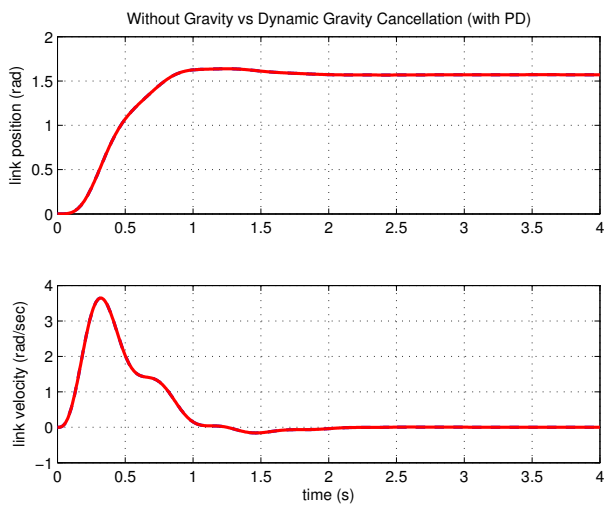

(a)
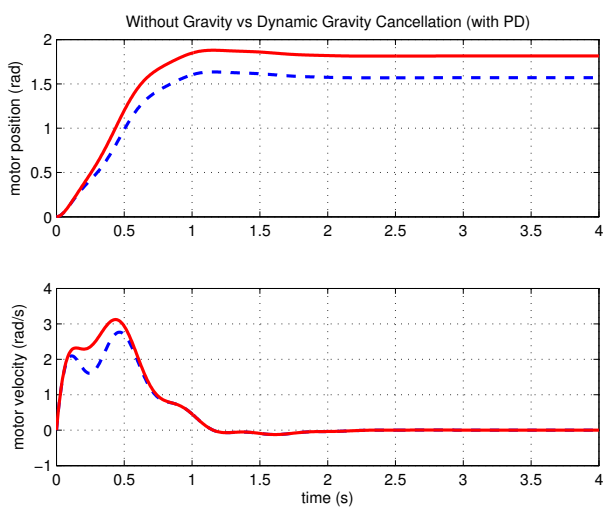

(b)

Fig. 1. Comparison of link variables (a) and motor variables (b) without gravity under PD control [dashed, blue] and with gravity under the PDtype control law with dynamic cancellation (16-17) [solid, red], when the stiffness is $K=100$

different evolution in the two cases (Fig. 1(b)), due to the need to charge the elastic joint for dynamically balancing gravity on the link. Figure 2(a) compares the total control torque in the two cases. The applied torque is different, since it vanishes at steady state when gravity is absent whereas it provides the static gravity torque at the goal in the other case. The contributions to the total torque $\tau_{g}+\tau_{0}$ are shown in Fig. 2(b), namely the PD term $\left(\tau_{0}\right)$, the static gravity term $g(q)=m d g_{0} \sin q$, and the remaining dynamic part of the gravity cancellation term $\left(\tau_{g}-g(q)\right)$. The PD term of the proposed control law is exactly the same as the PD action in the absence of gravity.

The control law is able to regulate the link position even when the joint stiffness is extremely small. Figures 3-4 show the results for the same previous PD gains but with joint stiffness smaller than the maximum gradient of the gravity term: $K=20<24.525=m d g_{0}$ (the structural condition is violated). The behavior is still satisfactory, although the transient is longer. The larger overshoot and the small oscillation experienced by the link while approaching the goal are due to the poor transient performance achievable by a motor PD feedback (with the chosen gains) in the absence of gravity.

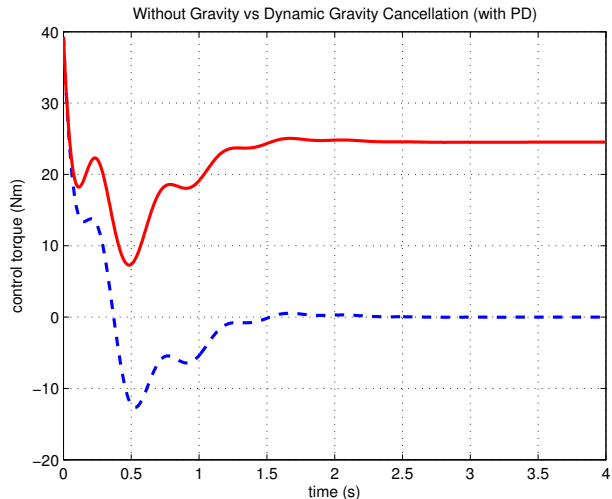

(a)

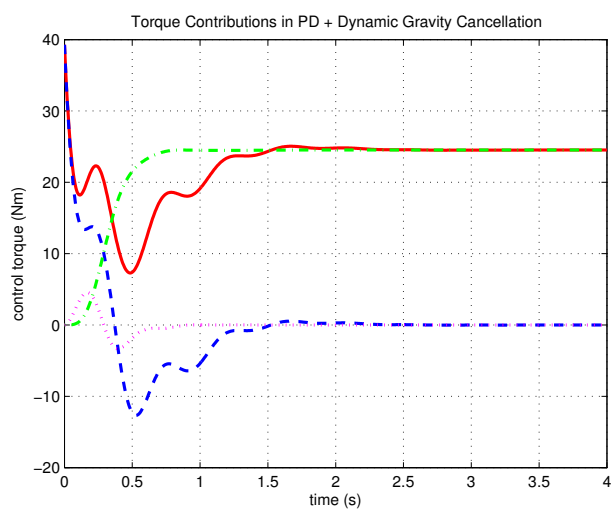

(b)

Fig. 2. Comparison of control torques (a) without gravity under PD control [dashed, blue] and with gravity under the PD-type control law with dynamic cancellation (16-17) [solid, red], when the stiffness is $K=100$. Torque contributions (b) in the proposed law: total [solid, red], PD term $\tau_{0}$ [dashed, blue], static gravity term $g(q)$ [dashed-dotted, green], dynamic cancellation term $\tau_{g}-g(q)$ [dotted, magenta]

\section{JoINTS WITH NONLINEAR FLEXIBILITY}

In this section, we extend the analysis of Sects. II-III to the case of transmissions with nonlinear flexibility [16]. For the sake of simplicity, only a single dof will be considered, but the generalization to multi-dof systems is straightforward.

For the flexible joint, a potential energy $U_{e}(\phi) \geq 0$ is associated to the joint deformation $\phi=q-\theta$ so that the flexibility torque $\tau_{e}=\partial U_{e} / \partial q=\tau_{e}(\phi)$ is a nonlinear function of $\phi$ and the stiffness $\sigma=\partial \tau_{e} / \partial q=\sigma(\phi)$ will be non-constant. Without loss of generality, we assume that $\tau_{e}(\phi)$ is a strictly increasing function for $\phi \in(-\infty,+\infty)$ and that $\tau_{e}(-\phi)=-\tau_{e}(\phi)$. The dynamic model of a single link moving under gravity and driven through this (nondissipative) flexible transmission is

$$
\begin{aligned}
M \ddot{q}+g(q)+\tau_{e}(\phi) & =0 \\
B \ddot{\theta}-\tau_{e}(\phi) & =\tau .
\end{aligned}
$$

\section{A. Gravity cancellation}

We define a feedback law $\tau=\tau\left(q, \theta, \dot{q}, \dot{\theta}, \tau_{0}\right)$ in (19) so as to match the behavior of the link variable $q$ with that of 

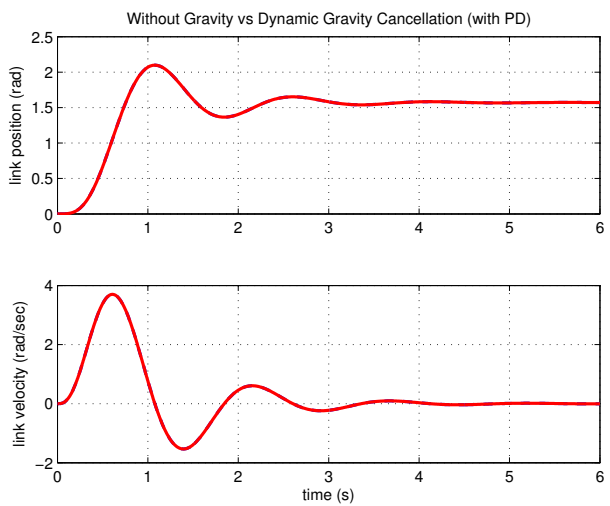

(a)
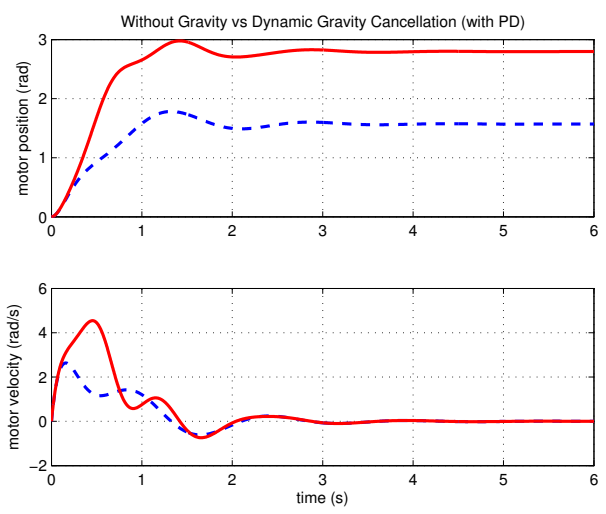

(b)

Fig. 3. Comparison of link variables (a) and motor variables (b) without gravity under PD control [dashed, blue] and with gravity under the PDtype control law with dynamic cancellation (16-17) [solid, red], when the stiffness is $K=20$

the link variable $q_{0}$ of the model without gravity

$$
\begin{aligned}
M \ddot{q}_{0}+\tau_{e}\left(\phi_{0}\right) & =0 \\
B \ddot{\theta}_{0}-\tau_{e}\left(\phi_{0}\right) & =\tau_{0} .
\end{aligned}
$$

It is easy to verify that both nonlinear systems (18-19) and (20-21) are feedback linearizable to a chain of four integrators, with the link positions and their first three time derivatives as linearizing coordinates. Therefore, the two systems are feedback equivalent, and the solution to our problem is found by imposing $q(t) \equiv q_{0}(t)$ for all $t \geq 0$. In particular, from $q^{[4]}=q_{0}^{[4]}$ we obtain

$$
\begin{aligned}
\tau= & g(q)+\frac{B}{\sigma(\phi)} \ddot{g}(q)+\frac{\sigma(\phi)-\sigma\left(\phi_{0}\right)}{\sigma(\phi)}(B+M) \ddot{q} \\
& +\frac{B}{\sigma(\phi)}\left(\frac{\partial \sigma(\phi)}{\partial \phi} \dot{\phi}^{2}-\frac{\partial \sigma\left(\phi_{0}\right)}{\partial \phi_{0}} \dot{\phi}_{0}^{2}\right)+\frac{\sigma\left(\phi_{0}\right)}{\sigma(\phi)} \tau_{0} \\
= & \tau_{g}+\alpha_{g} \tau_{0},
\end{aligned}
$$

where $\ddot{q}$ (to be used also in $\ddot{g}(q)$ ) is computed from (18) as

$$
\ddot{q}=-\frac{1}{M}\left(g(q)+\tau_{e}(\phi)\right) .
$$

Under (22), the link evolution of the gravity-loaded system will coincide with that of the gravity-free system, initialized

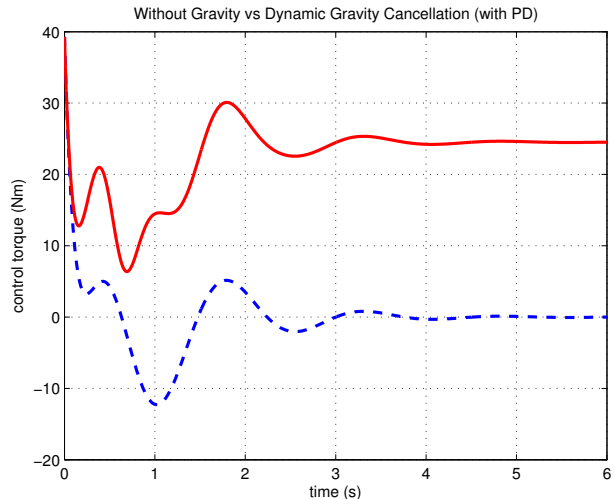

(a)

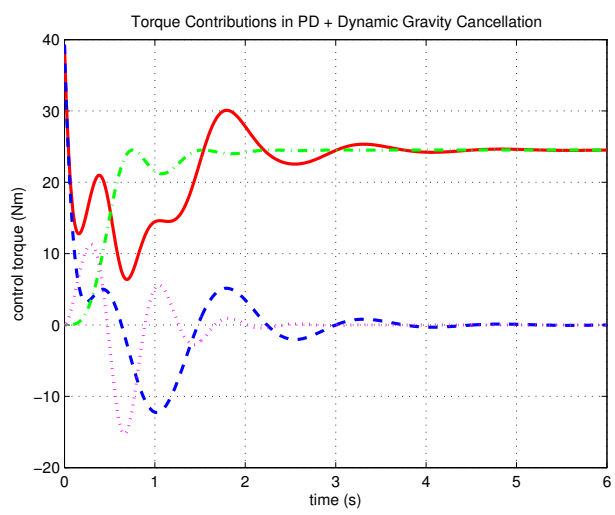

(b)

Fig. 4. Comparison of control torques (a) without gravity under PD control [dashed, blue] and with gravity under the PD-type control law with dynamic cancellation (16-17) [solid, red], when the stiffness is $K=20$. Torque contributions (b) in the proposed law: total [solid, red], PD term $\tau_{0}$ [dashed, blue], static gravity term $g(q)$ [dashed-dotted, green], dynamic cancellation term $\tau_{g}-g(q)$ [dotted, magenta]

at $t=0$ with the matching conditions as in (12). For a transmission with constant stiffness $\sigma=K$, the expression (22) collapses to (10-11). However, differently from the case of linear elasticity, the control law contains terms that require the knowledge of the deformation $\phi_{0}=q-\theta_{0}$, and of its rate $\dot{\phi}_{0}$, pertaining to the model without gravity. Also, the torque $\tau_{0}$ applied in the gravity-free case is now scaled by the factor $\alpha_{g}=\sigma\left(\phi_{0}\right) / \sigma(\phi)$.

The value $\phi_{0}$ to be used in (22) is obtained by comparing (20) with (18), under the identity $q(t) \equiv q_{0}(t)$. This gives the nonlinear equation

$$
\tau_{e}\left(\phi_{0}\right)=g(q)+\tau_{e}(\phi)=a(q, \theta) .
$$

Equation (23) needs to be solved numerically at each time $t \geq 0$, as a function of the current configuration of the gravity-loaded system. In some cases, a closed-form solution can be obtained. As a representative nonlinear example, consider a flexible joint transmission with associated potential given by $U_{e}=\frac{1}{2} K \phi^{2}+\frac{1}{4} K_{c} \phi^{4}$, with $K>0$ and $K_{c}>0$ [5]. The flexibility torque is a cubic function of $\phi$ and the stiffness 
has a quadratic dependence:

$$
\tau_{e}(\phi)=K \phi+K_{c} \phi^{3}, \quad \sigma(\phi)=K+3 K_{c} \phi^{2} .
$$

At a given $(q, \theta)$, equation (23) results in the cubic equation $K_{c} \phi_{0}^{3}+K \phi_{0}-a(q, \theta)=0$, which has always two complex roots and one real (positive or negative) root, thanks to the positivity of $K$ and $K_{c}$. The real root is given by

$$
\phi_{0}=\sqrt[3]{\frac{1}{2} \frac{a(q, \theta)}{K_{c}}+b(q, \theta)}+\sqrt[3]{\frac{1}{2} \frac{a(q, \theta)}{K_{c}}-b(q, \theta)},
$$

where $b(q, \theta)=\sqrt{\frac{1}{27}\left(\frac{K}{K_{c}}\right)^{3}+\frac{1}{4}\left(\frac{a(q, \theta)}{K_{c}}\right)^{2}}>0$.

Once $\phi_{0}$ has been found (numerically or analytically), the value of $\dot{\phi}_{0}$ in the control law (22) is obtained in closed form by time differentiation of (23) as

$$
\dot{\phi}_{0}=\frac{1}{\sigma\left(\phi_{0}\right)}\left(\sigma(\phi) \dot{\phi}+\frac{\partial g(q)}{\partial q} \dot{q}\right) \text {. }
$$

\section{B. Regulation control law}

For regulating the link position $q$ of system (18-19) to a desired $q_{d}$, we set $\tau=\tau_{g}+\tau_{0}$ with $\tau_{g}$ as in (22) and $\tau_{0}$ given by

$$
\tau_{0}=K_{P}\left(q_{d}-\theta_{0}\right)-K_{D} \dot{\theta}_{0},
$$

where $K_{P}>0$ and $K_{D}>0$. Global asymptotic stability can be shown without any further assumption by using the Lyapunov candidate

$$
V=\frac{1}{2} M \dot{q}^{2}+\frac{1}{2} B \dot{\theta}_{0}^{2}+\frac{1}{2} K_{P}\left(q_{d}-\theta_{0}\right)^{2}+U_{e}\left(\phi_{0}\right) \geq 0,
$$

which is the Lyapunov function for the gravity-free system (20-21) under (26), In eqs. (26) and (27), we need to use $\theta_{0}=q-\phi_{0}$ and $\dot{\theta}_{0}=\dot{q}-\dot{\phi}_{0}$, where $\phi_{0}$ and $\dot{\phi}_{0}$ are obtained from (23) and (25), respectively. The proof follows along the same lines of Theorem 1 (and it can be easily extended to the multi-dof case).

\section{CONCLUSIONS}

We have presented a new regulation approach for robot manipulators having flexible joints. The control law consists of two terms. The first term achieves dynamic cancellation of gravity effects acting on the link motion and is based on the feedback equivalence principle. It requires the on-line computation of inertial terms, but is otherwise much simpler than a complete feedback linearization law. The second term is a PD-type feedback from the error on the motor position of the associated gravity-free model, which is nonlinearly related to the motor position of the system at hand.

Global asymptotic stabilization of the desired link position is obtained under the weakest possible assumptions of positive definiteness of the PD gains and of the robot joint stiffness. The proposed solution is obtained in closed form for robots with elastic joints (of constant stiffness) or, more in general, using a simple numerical technique in the presence of nonlinear flexible joints (of variable stiffness).

We are currently extending this regulation approach to the case of robots with variable and independently actuated nonlinear stiffness [6], [7], where the link position as well as the device stiffness need to be asymptotically stabilized to a desired constant value. Initial results on dynamic cancellation of gravity in VSA-based robots can be found in [17]. Indeed, the idea of exact gravity cancellation can be used also for developing simpler trajectory tracking control laws, another topic of further research.

Gravity cancellation in compliant manipulators finds a useful application in safe physical human-robot interaction. When unexpected collisions occur during motion, the robot should react as soon as the impact is detected. By realizing a zero-gravity condition for the robot links, a simpler, physicalbased torque reaction strategy can be designed for the control term $\tau_{0}$, as opposed to the reaction based on feedback linearization control proposed in [18], so that the robot rapidly flees away from the collision area in a gravityunbiased fashion.

\section{ACKNOWLEDGMENTS}

This work has been funded by the MIUR project PRIN 2007 SICURA.

\section{APPENDIX \\ PROOF OF THEOREM 1}

The proof is based on Lyapunov analysis and on LaSalle theorem. For the closed-loop system (1-2) under (14-15), we show first that there is a unique equilibrium state, i.e., an equilibrium configuration $\left(\boldsymbol{q}^{e}, \boldsymbol{\theta}^{e}\right)$ with zero velocities $\dot{\boldsymbol{q}}$ and $\dot{\boldsymbol{\theta}}$. Setting $\ddot{\boldsymbol{q}}=\ddot{\boldsymbol{\theta}}=\mathbf{0}$ in the closed-loop equations, any equilibrium configuration should satisfy

$$
\begin{aligned}
\boldsymbol{g}\left(\boldsymbol{q}^{e}\right)+\boldsymbol{K}\left(\boldsymbol{q}^{e}-\boldsymbol{\theta}^{e}\right) & =\mathbf{0} \\
\boldsymbol{K}\left(\boldsymbol{q}^{e}-\boldsymbol{\theta}^{e}\right)+\boldsymbol{g}\left(\boldsymbol{q}^{e}\right)+\boldsymbol{K}_{P}\left(\boldsymbol{q}_{d}-\boldsymbol{\theta}^{e}+\boldsymbol{K}^{-1} \boldsymbol{g}\left(\boldsymbol{q}^{e}\right)\right) & =\mathbf{0} .
\end{aligned}
$$

Subtracting the two equations gives $\boldsymbol{\theta}^{e}=\boldsymbol{q}_{d}+\boldsymbol{K}^{-1} \boldsymbol{g}\left(\boldsymbol{q}^{e}\right)$, while the first equation yields $\boldsymbol{\theta}^{e}=\boldsymbol{q}^{e}+\boldsymbol{K}^{-1} \boldsymbol{g}\left(\boldsymbol{q}^{e}\right)$. By comparison, it follows that

$$
\boldsymbol{q}^{e}=\boldsymbol{q}_{d}, \quad \boldsymbol{\theta}^{e}=\boldsymbol{q}_{d}+\boldsymbol{K}^{-1} \boldsymbol{g}\left(\boldsymbol{q}_{d}\right)=\boldsymbol{\theta}_{d}
$$

and thus $\left(\boldsymbol{q}_{d}, \boldsymbol{\theta}_{d}\right)$ is the unique equilibrium configuration.

Without loss of generality, the positive-definite gain matrices $\boldsymbol{K}_{P}$ and $\boldsymbol{K}_{D}$ are chosen symmetric. Let a Lyapunov candidate be defined as

$$
\begin{aligned}
V= & \frac{1}{2}\left(\dot{\boldsymbol{q}}^{T} \boldsymbol{M}(\boldsymbol{q}) \dot{\boldsymbol{q}}+\left(\dot{\boldsymbol{\theta}}-\boldsymbol{K}^{-1} \dot{\boldsymbol{g}}(\boldsymbol{q})\right)^{T} \boldsymbol{B}\left(\dot{\boldsymbol{\theta}}-\boldsymbol{K}^{-1} \dot{\boldsymbol{g}}(\boldsymbol{q})\right)\right. \\
& +\left(\boldsymbol{q}-\boldsymbol{\theta}+\boldsymbol{K}^{-1} \boldsymbol{g}(\boldsymbol{q})\right)^{T} \boldsymbol{K}\left(\boldsymbol{q}-\boldsymbol{\theta}+\boldsymbol{K}^{-1} \boldsymbol{g}(\boldsymbol{q})\right) \\
& \left.+\left(\boldsymbol{q}_{d}-\boldsymbol{\theta}+\boldsymbol{K}^{-1} \boldsymbol{g}(\boldsymbol{q})\right)^{T} \boldsymbol{K}_{P}\left(\boldsymbol{q}_{d}-\boldsymbol{\theta}+\boldsymbol{K}^{-1} \boldsymbol{g}(\boldsymbol{q})\right)\right) .
\end{aligned}
$$

As sum of positive definite terms in a quadratic structure, $V$ is positive definite. Moreover, $V=0$ if and only if

$$
\dot{\boldsymbol{q}}=\mathbf{0}, \quad \dot{\boldsymbol{\theta}}-\boldsymbol{K}^{-1} \frac{\partial \boldsymbol{g}(\boldsymbol{q})}{\partial \boldsymbol{q}} \dot{\boldsymbol{q}}=\mathbf{0} \quad \Rightarrow \quad \dot{\boldsymbol{\theta}}=\mathbf{0}
$$

and

$$
\left.\begin{array}{rl}
\boldsymbol{q}-\boldsymbol{\theta}+\boldsymbol{K}^{-1} \boldsymbol{g}(\boldsymbol{q}) & =\mathbf{0} \\
\boldsymbol{q}_{d}-\boldsymbol{\theta}+\boldsymbol{K}^{-1} \boldsymbol{g}(\boldsymbol{q}) & =\mathbf{0}
\end{array}\right\} \Rightarrow\left\{\begin{array}{l}
\boldsymbol{q}=\boldsymbol{q}_{d} \\
\boldsymbol{\theta}=\boldsymbol{q}_{d}+\boldsymbol{K}^{-1} \boldsymbol{g}\left(\boldsymbol{q}_{d}\right) .
\end{array}\right.
$$


Therefore, the desired state is the unique minimum of $V$. Dropping dependencies for compactness, the time derivative of $V$ is

$$
\begin{aligned}
\dot{V}= & \dot{\boldsymbol{q}}^{T} \boldsymbol{M} \ddot{\boldsymbol{q}}+\frac{1}{2} \dot{\boldsymbol{q}}^{T} \dot{\boldsymbol{M}} \dot{\boldsymbol{q}}+\left(\dot{\boldsymbol{\theta}}-\boldsymbol{K}^{-1} \dot{\boldsymbol{g}}\right)^{T} \boldsymbol{B}\left(\ddot{\boldsymbol{\theta}}-\boldsymbol{K}^{-1} \ddot{\boldsymbol{g}}\right) \\
& +\left(\boldsymbol{q}-\boldsymbol{\theta}+\boldsymbol{K}^{-1} \boldsymbol{g}\right)^{T} \boldsymbol{K}\left(\dot{\boldsymbol{q}}-\dot{\boldsymbol{\theta}}+\boldsymbol{K}^{-1} \dot{\boldsymbol{g}}\right) \\
& -\left(\boldsymbol{q}_{d}-\boldsymbol{\theta}+\boldsymbol{K}^{-1} \boldsymbol{g}\right)^{T} \boldsymbol{K}_{P}\left(\dot{\boldsymbol{\theta}}-\boldsymbol{K}^{-1} \dot{\boldsymbol{g}}\right) .
\end{aligned}
$$

The closed-loop equations (1-2) with (14-15) can be conveniently rewritten in the form

$$
\begin{aligned}
& \boldsymbol{M} \ddot{\boldsymbol{q}}=\boldsymbol{K}(\boldsymbol{\theta}-\boldsymbol{q})-\boldsymbol{S} \dot{\boldsymbol{q}}-\boldsymbol{g} \\
& \boldsymbol{B}\left(\ddot{\boldsymbol{\theta}}-\boldsymbol{K}^{-1} \ddot{\boldsymbol{g}}\right)=\boldsymbol{K}(\boldsymbol{q}-\boldsymbol{\theta})+\boldsymbol{g} \\
& \quad+\boldsymbol{K}_{P}\left(\boldsymbol{q}_{d}-\boldsymbol{\theta}+\boldsymbol{K}^{-1} \boldsymbol{g}\right)-\boldsymbol{K}_{D}\left(\dot{\boldsymbol{\theta}}-\boldsymbol{K}^{-1} \dot{\boldsymbol{g}}\right) .
\end{aligned}
$$

Substituting these into the expression of $\dot{V}$ and simplifying terms yields

$$
\begin{aligned}
\dot{V}= & \dot{\boldsymbol{q}}^{T}\left(\boldsymbol{K}(\boldsymbol{\theta}-\boldsymbol{q})+\frac{1}{2}(\dot{\boldsymbol{M}}-2 \boldsymbol{S}) \dot{\boldsymbol{q}}-\boldsymbol{g}\right) \\
+ & \left(\dot{\boldsymbol{\theta}}-\boldsymbol{K}^{-1} \dot{\boldsymbol{g}}\right)^{T}(\boldsymbol{K}(\boldsymbol{q}-\boldsymbol{\theta})+\boldsymbol{g} \\
& \left.\quad+\boldsymbol{K}_{P}\left(\boldsymbol{q}_{d}-\boldsymbol{\theta}+\boldsymbol{K}^{-1} \boldsymbol{g}\right)-\boldsymbol{K}_{D}\left(\dot{\boldsymbol{\theta}}-\boldsymbol{K}^{-1} \dot{\boldsymbol{g}}\right)\right) \\
& +(\boldsymbol{K}(\boldsymbol{q}-\boldsymbol{\theta})+\boldsymbol{g})^{T}\left(\dot{\boldsymbol{q}}-\dot{\boldsymbol{\theta}}+\boldsymbol{K}^{-1} \dot{\boldsymbol{g}}\right) \\
& -\left(\boldsymbol{q}_{d}-\boldsymbol{\theta}+\boldsymbol{K}^{-1} \boldsymbol{g}\right)^{T} \boldsymbol{K}_{P}\left(\dot{\boldsymbol{\theta}}-\boldsymbol{K}^{-1} \dot{\boldsymbol{g}}\right) \\
= & -\left(\dot{\boldsymbol{\theta}}-\boldsymbol{K}^{-1} \dot{\boldsymbol{g}}\right)^{T} \boldsymbol{K}_{D}\left(\dot{\boldsymbol{\theta}}-\boldsymbol{K}^{-1} \dot{\boldsymbol{g}}\right) \leq 0,
\end{aligned}
$$

where the energy-based relation $\dot{\boldsymbol{q}}^{T}(\dot{\boldsymbol{M}}-2 \boldsymbol{S}) \dot{\boldsymbol{q}}=\mathbf{0}$ has been used. Thus, it is

$$
\dot{V}=0 \quad \Leftrightarrow \quad \dot{\boldsymbol{g}}(\boldsymbol{q})-\boldsymbol{K} \dot{\boldsymbol{\theta}}=\mathbf{0} .
$$

We proceed next using LaSalle arguments. The desired state satisfies indeed $\dot{V}=0$, and thus is invariant with respect to the set of states where $\dot{V}=0$ (and gives $V(t) \equiv 0$ ). We should verify that there are no other system trajectories that are invariant with respect to the set defined by $\dot{V}=0$. When $\dot{V}=0$, note first that

$$
\frac{d}{d t}(\boldsymbol{g}(\boldsymbol{q})-\boldsymbol{K} \boldsymbol{\theta})=\mathbf{0} \quad \Rightarrow \quad \boldsymbol{g}(\boldsymbol{q})-\boldsymbol{K} \boldsymbol{\theta}=\boldsymbol{k}_{1},
$$

where $\boldsymbol{k}_{1}$ is a suitable constant vector. Moreover, the model equation (2) with $\tau$ as in (14-15) becomes

$$
\begin{aligned}
& \boldsymbol{B} \ddot{\boldsymbol{\theta}}+\boldsymbol{K}(\boldsymbol{\theta}-\boldsymbol{q})= \\
& \quad \boldsymbol{g}(\boldsymbol{q})+\boldsymbol{B} \boldsymbol{K}^{-1} \ddot{\boldsymbol{g}}(\boldsymbol{q})+\boldsymbol{K}_{P}\left(\boldsymbol{q}_{d}-\boldsymbol{\theta}+\boldsymbol{K}^{-1} \boldsymbol{g}(\boldsymbol{q})\right),
\end{aligned}
$$

or, by simple manipulation,

$$
\begin{aligned}
& \boldsymbol{B} \boldsymbol{K}^{-1} \frac{d}{d t}(\boldsymbol{K} \dot{\boldsymbol{\theta}}-\dot{\boldsymbol{g}}(\boldsymbol{q}))= \\
& \quad\left(\boldsymbol{I}+\boldsymbol{K}_{P} \boldsymbol{K}^{-1}\right)[\boldsymbol{g}(\boldsymbol{q})-\boldsymbol{K} \boldsymbol{\theta}]+\boldsymbol{K} \boldsymbol{q}+\boldsymbol{K}_{P} \boldsymbol{q}_{d} .
\end{aligned}
$$

For a closed-loop system trajectory to remain in the set of states such that $\dot{V}=0$, the left-hand side of this equation must be zero. Since the term in square brackets on the righthand side is constant, it follows that

$$
\boldsymbol{K} \boldsymbol{q}+\boldsymbol{K}_{P} \boldsymbol{q}_{d}=\boldsymbol{k}_{2},
$$

where $\boldsymbol{k}_{2}$ is a suitable constant vector. Hence, $\boldsymbol{q}$ is constant and, as a consequence, $\boldsymbol{\theta}$ is also a constant. Thus, $\dot{\boldsymbol{q}}=\dot{\boldsymbol{\theta}}=\mathbf{0}$ and the only invariant trajectory compatible with $\dot{V}=0$ is an equilibrium state. Since the desired state $\boldsymbol{q}=\boldsymbol{q}_{d}, \boldsymbol{\theta}=\boldsymbol{\theta}_{d}$, with $\dot{\boldsymbol{q}}=\dot{\boldsymbol{\theta}}=\mathbf{0}$, is the unique closed-loop equilibrium state, then it is globally asymptotically stable thanks to LaSalle theorem. This completes the proof.

\section{REFERENCES}

[1] B. Siciliano, L. Sciavicco, L. Villani, and G. Oriolo, Robotics: Modeling, Planning and Control, 3rd ed. London: Springer, 2008.

[2] J. Heinzmann and A. Zelinsky, "Quantitative safety guarantees for physical human-robot interaction," Int. J. of Robotics Research, vol. 22, no. 7/8, pp. 479-504, 2003.

[3] A. De Luca, A. Albu-Schäffer, S. Haddadin, and G. Hirzinger, "Collision detection and safe reaction with the DLR-III lightweight robo arm," in Proc. IEEE/RSJ Int. Conf. on Intelligent Robots and Systems, 2006, pp. 1623-1630.

[4] G. Hirzinger, A. Albu-Schäffer, M. Hähnle, I. Schaefer, and N. Sporer, "On a new generation of torque controlled light-weight robots," in Proc. IEEE Int. Conf. on Robotics and Automation, 2001, pp. 33563363.

[5] T. D. Tuttle and W. P. Seering, "A nonlinear model of a harmonic drive gear transmission," IEEE Trans. on Robotics and Automation, vol. 12 , no. 3, pp. 368-374, 1996.

[6] A. Bicchi and G. Tonietti, "Fast and soft arm tactics: Dealing with the safety-performance trade-off in robot arms design and control," IEEE Robotics and Automation Mag., vol. 11, no. 2, pp. 22-33, 2004.

[7] S. Wolf and G. Hirzinger, "A new variable stiffness design: Matching requirements of the next robot generation," in Proc. IEEE Int. Conf. on Robotics and Automation, 2008, pp. 1741-1746.

[8] M. W. Spong, "Modeling and control of elastic joint robots," ASME J. of Dynamic Systems, Measurement, and Control, vol. 109, no. 4, pp. 310-319, 1987.

[9] P. Tomei, "A simple PD controller for robots with elastic joints," IEEE Trans. on Automatic Control, vol. 36, no. 10, pp. 1208-1213, 1991.

[10] A. De Luca, B. Siciliano, and L. Zollo, "PD control with online gravity compensation for robots with elastic joints: Theory and experiments," Automatica, vol. 41, no. 10, pp. 1809-1819, 2005.

[11] A. Kugi, C. Ott, A. Albu-Schäffer, and G. Hirzinger, "On the passivitybased impedance control of flexible joint robots," IEEE Trans. on Robotics, vol. 24, no. 2, pp. 416-429, 2008

[12] S. Haddadin, A. Albu-Schäffer, A. De Luca, and G. Hirzinger, "Collision detection and reaction: A contribution to safe physical humanrobot interaction," in Proc. IEEE/RSJ Int. Conf. on Intelligent Robots and Systems, 2008, pp. 3356-3363.

[13] A. Isidori, Nonlinear Control Systems, 3rd ed. Springer, 1995.

[14] A. De Luca and W. Book, "Robots with flexible elements," in Springer Handbook of Robotics, B. Siciliano and O. Khatib, Eds. Springer, 2008, pp. 287-319.

[15] C. Ott, Cartesian Impedance Control of Redundant and FlexibleJoint Robots, Springer Tracts in Advanced Robotics (STAR), Vol. 49. Springer, 2008

[16] N. Kircanski and A. Goldenberg, "An experimental study of nonlinear stiffness, hysteresis, and friction effects in robot joints with harmonic drives and torque sensors," Int. J. of Robotics Research, vol. 16, no. 2, pp. 214-239, 1997.

[17] A. De Luca and F. Flacco, "Dynamic gravity cancellation in robots with flexible transmissions," in 49th IEEE Conf. on Decision and Control, 2010, pp. 288-295.

[18] A. De Luca, F. Flacco, A. Bicchi, and R. Schiavi, "Nonlinear decoupled motion-stiffness control and collision detection/reaction for the VSA-II variable stiffness device," in Proc. IEEE/RSJ Int. Conf. on Intelligent Robots and Systems, 2009, pp. 5487-5494. 